\title{
Über dichroitisch angefärbte Polarisatoren
}

\author{
Von H. Scherer \\ Aus dem Physikalischen Institut der Justus-Liebig-Hochschule, Gießen \\ (Z. Naturforschg. 6 a. 440-445 [1951]; eingegangen am 28. April 1951)

\begin{abstract}
Gereckte und gefärbte Kolloidfolien zeigen sehr oft einen starken Dichroismus. Dieser wird im Bereich von 250 bis $500 \mathrm{~m} \mu$ untersucht und auf eine gerichtete Adsorption zurückgeführt. Ferner wird die Möglichkeit gezeigt, Farbstoffpolarisatoren auch für ultraviolettes Licht zu bauen.
\end{abstract}

S owohl die Theorie von A $m b$ ro $n^{1}{ }^{1}$ als auch die von $\mathrm{B} \mathrm{r} \mathrm{a} \mathrm{u} \mathrm{n}{ }^{2}$ sind nicht in der Lage, alle Erscheinungen auf dem Gebiet dichroitischer Färbungen zu erklären. Die zwangloseste Deutung gibt die Adsorptionstheorie von $\mathrm{Fr} \mathrm{e} \mathrm{y}{ }^{3}$. Nach ihr lassen sich alle Beobachtungen des Dichroismus gefärbter Folien verstehen, wenn man sie mit Ambronn auf die optischen Eigenschaften der eingelagerten Stoffe zurückführt und sich die Einlagerung als eine gerichtete Adsorption vorstellt. Ambronn, Braun und Frey kamen zu ihren Theorien auf Grund qualitativer Beobachtungen. Im folgenden werden die Ergebnisse quantitativer Messungen wiedergegeben. Der zu untersuchende Spektralbereich wird in das nahe Ultraviolett verlegt und somit gleichzeitig die Möglichkeit gezeigt, derartige Polarisatoren auch für das ultraviolette Licht zu bauen.

Für homogen absorbierende Medien ist die Lichtabsorption gegeben durch

oder

$$
\frac{J}{J_{0}}=D=10^{-E}
$$

$$
E=\log \frac{J_{0}}{J}=\log \frac{1}{D}
$$

( $E=$ Absorption, $D=$ Durchlässigkeit).

Dichroitische Medien sind dadurch gekennzeichnet, daß sie für zwei senkrecht zueinander polarisierte einfallende Strahlen verschiedene Absorption zeigen. Für eine vollständige Beschreibung solcher Medien werden also Absorptionskoeffizienten $E_{\|}$ und $E_{\perp}$ eingeführt. Der mathematische Ausdruck für Dichroismus ist dann

$$
\Delta E=E_{\|}-E_{\perp} .
$$

1 H. A m b r o n n, Ann. Physik 34, 344 [1888].

2 F. B r a u n, Ann. Physik 16, 1, 238 [1905].

3 A. F r e y, Naturwiss. 13, 403 [1925].
Im folgenden soll unter $E_{\|}$die Absorption verstanden werden für Licht, dessen elektrischer Vektor parallel der Reckrichtung der gefärbten Folie schwingt. Analoges gilt für $E_{1}$.

Bei linear dichroitischen Folien kann

$$
E=\log \frac{J_{0}}{J}=\log \frac{1}{D_{\mathrm{N}}}
$$

$\left(D_{N}=\right.$ Durchlässigkeit für natürliches Licht) auf zwei Arten definiert und gemessen werden. Es ist entweder die Absorption für natürliches oder zirkularpolarisiertes Licht, oder aber es ist die Absorption für linear polarisiertes Licht, dessen Schwingungsrichtung unter $45^{\circ}$ zur Reckrichtung der Folie geneigt ist.

\section{Eigenschaften und Dichroismus ungefärbter Folien}

Die in dieser Arbeit vorwiegend verwandten Polyvinylalkoholfolien waren von der Fa. Dr. A lex a nder W a c k e r, München, freundlichst zur Verfügung gestellt. Das Material ist farblos und durchsichtig und die Filme sind optisch isotrop. Ihre Dicke beträgt ungefähr 50 bis $60 \mu$. Bei überelastischer Dehnung werden die Folien plötzlich milchig trübe und verhalten sich im Polarisationsmikroskop wie ein optisch einachsiger Körper mit positiver Doppelbrechung, dessen optische Achse in die Zugrichtung fällt. Stärkere Doppelbrechung wurde durch Dehnen im feuchten Zustand erzielt. Hierbei konnten Dehnungen von durchschnittlich 500-600\% erreicht werden, ohne daß eine Trübung auftrat und die Folie riß. Zeugnis von dem orientierten Zustand dieses linear hochpolymeren gedehnten Materials geben die mechanischen und optischen Anisotropien. Die Folien besitzen senkrecht zur Reckrichtung eine wesentlich größere Zerreißfestigkeit als in paralleler Richtung. Ferner sind sie positiv doppelbrechend. Nach dem allgemeinen $\mathrm{Zu}-$ sammenhang von Dispersion und Absorption müssen 


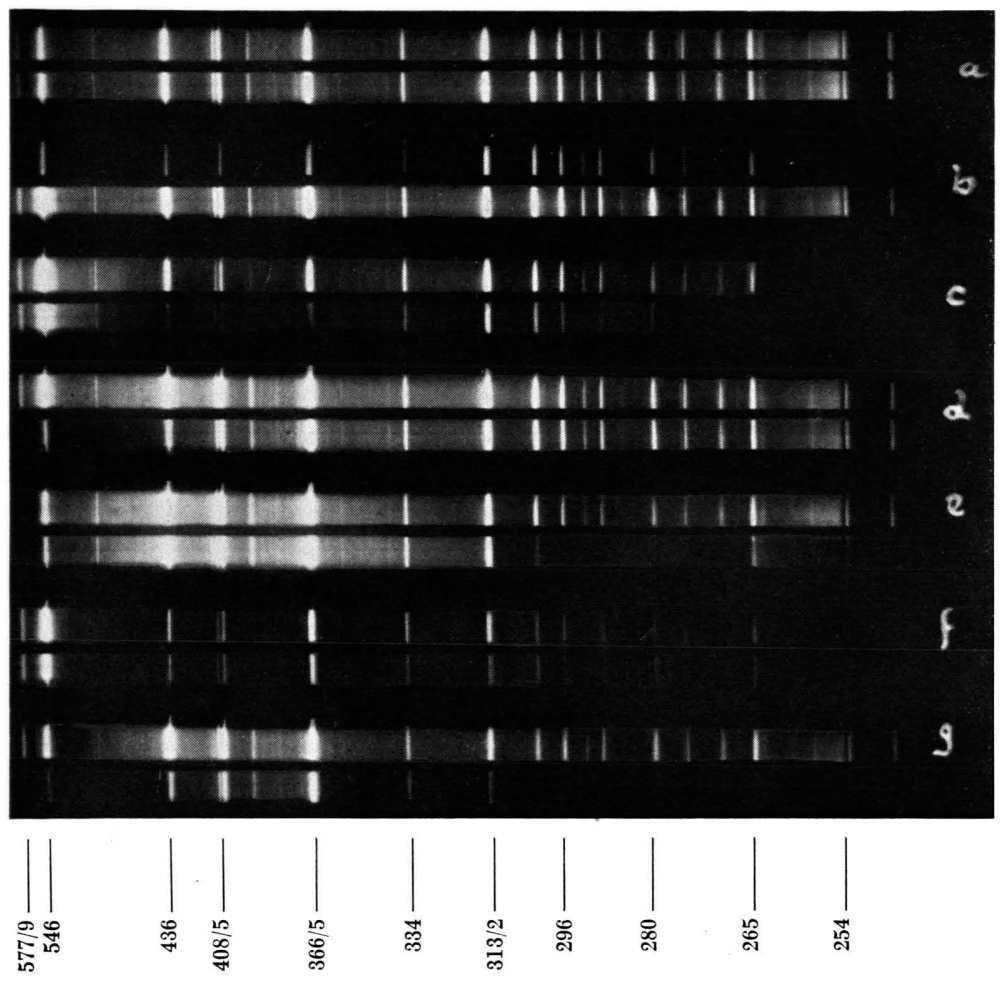

Wellenlängen in mu

a) Hg-Spektrum ohne Polarisationsfilter

b) mit Polarisationsfilter, Farbstoff: Siriuslichtbraun

c) mit Polarisationsfilter, Farbstoff: Thiazolgelb

d) mit Polarisationsfilter, Farbstoff : Diazurin B

e) mit Polarisationsfilter, Farbstoff: Methylenblau BGx

f) mit Polarisationsfilter, Farbstoff: Chrysoidin G

g) mit Polarisationsfilter, Farbstoff: Diazoblauschwarz RS

Abb. 1. Spektralaufnahmen von Folien, die mit verschiedenen Farbstoffen angefärbt wurden. 


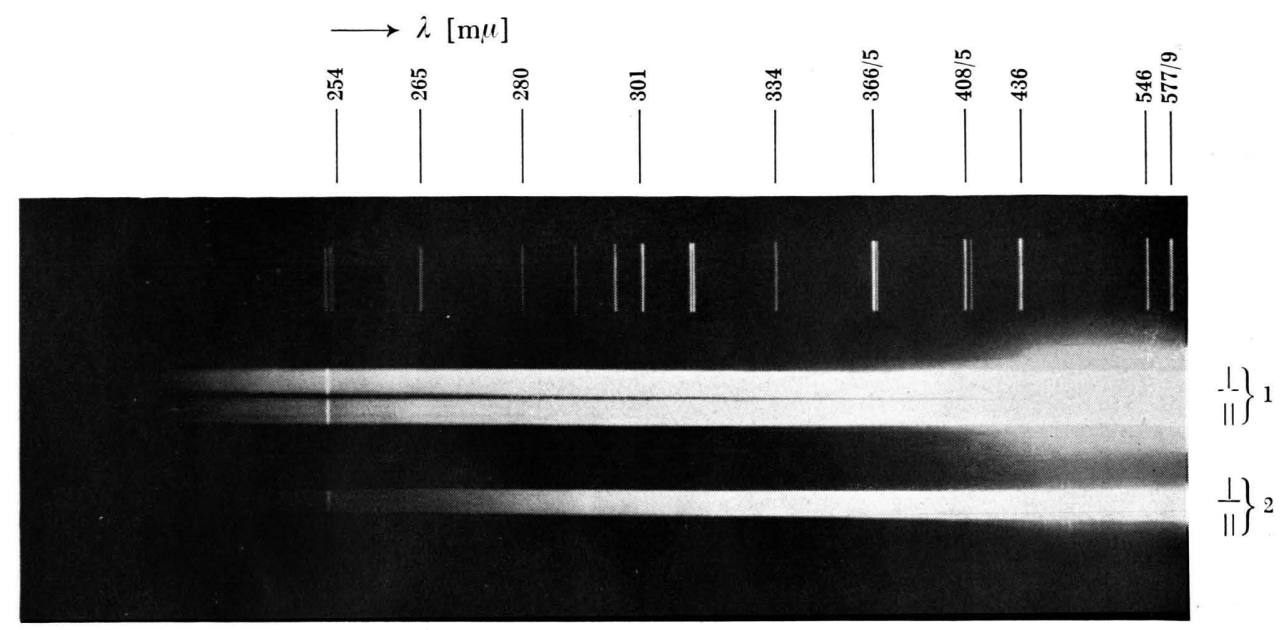

Abb. 13. Absorptionsspektrum eines UV-Polarisationsfilters. Hergestellt durch Färben gereckter Kolloidfolien mit verschiedenen Farbstoffen. 1. Xe-Kontinuum ohne Filter; 2. Xe-Kontinuum mit Polarisationsfilter. $(\perp=$ senkrecht zur Reckrichtung schwingendes Licht); $\|=$ parallel zur Reckrichtung schwingendes Licht).

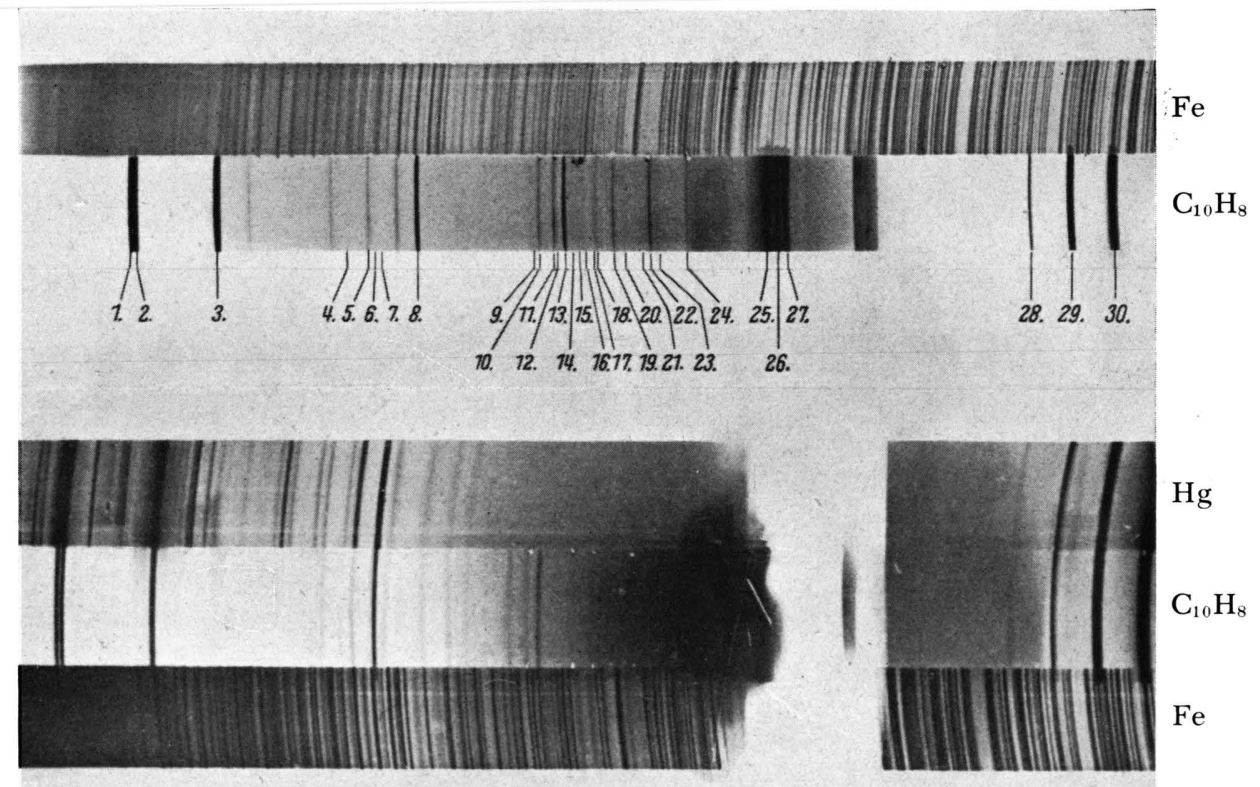

Abb. 3. Raman-Spektrum des festen Naphthalins, oben mit, unten ohne sekund. Reflexionsfilter. 
solche Folien aber im Gebiet ihrer Eigenabsorption Dichroismus zeigen. Derselbe konnte beim Beginn der Eigenabsorption der Folie gemessen werden (Abb. 1). Er ist nur gering und hat für die Herstellung von Folarisatoren keine Bedeutung.

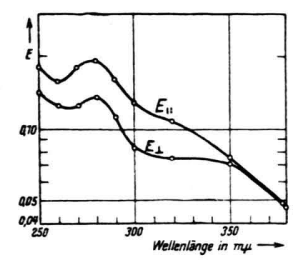

Abb. 1. Eigenabsorption einer ungefärbten gereckten Polyvinylalkoholfolie (Dicke $33 \mu$ ) ohne Reflexionsverlust.

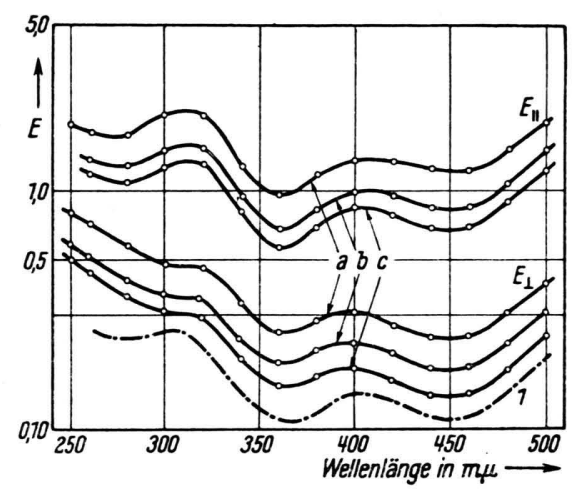

Abb. 2. Absorptionsspektrum gereckter und gefärbter Folien. Farbstoff: Diazobrillantscharlach B A. a) 10 Min. gefärbt, b) 5 Min. gefärbt, c) 2 Min. gefärbt. 1. Absorption desselben Farbstoffes in 10-2-mol. Lösung.

\section{Farbstoffpolarisatoren}

Orientierte Polyvinylalkoholfolien zeigen nach Anfärbung mit substantiven Farben allgemein stark positiven Dichroismus. Es wurden mit etwa 300 Farbstoffen Anfärbungen vorgenommen, die alle zu den gleichen Ergebnissen führten.

Vergleicht man die Absorptionsspektren der Farbstofflösung mit denen der angefärbten Folien für natürliches Licht, so treten nur unwesentliche Änderungen auf. In Abb. 2 und 3 sind die Absorptionsspektren der Farbstofflösungen mit eingezeichnet. F'erner ist in Abb. 3 die Absorption $E_{\mathrm{N}}$ der angefärbten Folie für natürliches Licht aus den Werten $E_{\|}$ und $E_{\perp}$ errechnet.

$$
\begin{aligned}
E_{\mathrm{N}} & =\log \frac{1}{D_{\mathrm{N}}} \\
& =\log \frac{2}{D_{\|}+D_{\perp}} \\
& =\log 2+E_{\left(D_{\|}+D_{\perp}\right)} .
\end{aligned}
$$

Die Kurven haben angenähert gleichen Verlauf, was in der logarithmischen Darstellung besagt, daß der Charakter der Lichtabsorption im adsorbierten $\mathrm{Zu}$ stand und in Lösung annähernd gleich ist.

Jedoch können bei adsorbierten Molekülen in den beiden ausgezeichneten Absorptionsrichtungen Banden auftreten, die nur einer Polarisationsrichtung angehören und die im Spektrum der Lösungen nur schwach erscheinen. Diese Banden sind zweifellos Oscillatoren zugeordnet, die im Molekül feste Lagen haben.

In den angefärbten Polyvinylalkoholfolien kommt

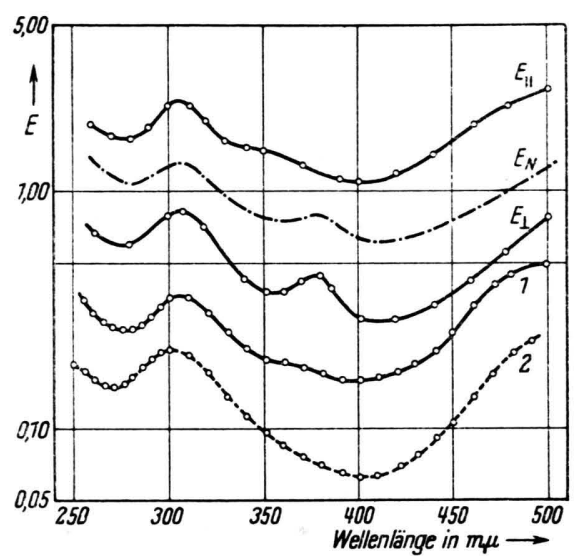

Abb. 3. Absorptionsspektrum einer Anfärbung mit Brillantponceau 4 R. 1 und 2 Spektren des Farbstoffes in wäßriger Lösung (Konz. $=10^{-2}$ und $10^{-5} \mathrm{~mol}$.); $E_{\mathrm{N}}=$ errechnet nach (2).

die Anisotropie der Lichtabsorption ${ }^{4}$ durch orientierte Adsorption von Farbstoffmolekülen an den Makromolekülen des Folienmaterials zustande, die durch Dehnung eine Vorzugsrichtung erhalten haben. Es läßt sich so ohne weiteres das Auftreten verschiedener Absorptionsbanden in den einzelnen Schwingungsrichtungen verstehen.

An dieser Stelle muß die Eigenschaft der Farbstoffe erwähnt werden, in konzentrierten Lösungen leicht größere Farbstoffaggregate (Kolloide) zu bilden. Diese Aggregatbildung wird auch sehr oft bei der Adsorption an Grenzflächen beobachtet und muß dann dafür verantwortlich gemacht werden, daß die $E_{\mathrm{N}}$-Kurve einer angefärbten Folie mehr der E-Kurve konzentrierter Lösungen entspricht. Vgl. Abb. 3 die Kurven: 1,2 und $E_{\mathrm{N}}$.

Die Absorptionsbanden einzelner Farbstoffe sind oft zu schmal, um größere dichroitische Bereiche zu

4 G. S c heibe, St. H a r twig u. R. M ülle r, Z. Elektrochem. angew. physik. Chem. 49, 372 [1943]. 
liefern. Es ist daher zweckmäßig, beim Bau von Polarisatoren Farbstoffgemische zu verwerten und diese so abzustimmen, daß die sich ergebenden Absorptionskurven nahezu horizontal verlaufen. In Abb. 4 zeigen die ausgezogenen Kurven die Absorption einer mit Siriuslichtbraun G angefärbten Folie. Eine andere Folie gleicher Dicke wurde mit Siriuslichtblau $3 \mathrm{RL}$ angefärbt und die gemessene Absorption (gestrichelt) in dasselbe Koordinatennetz eingetragen. Wie sich aus den Kurven entnehmen läßt, waren die Farbstoffe so gewählt, daß die Absorptionsmaxima des einen auf die Minima des anderen fielen. Man kann nun, um einen horizontalen Verlauf der dichroitischen Absorption $\mathrm{zu}$ erzielen, beide Filter parallel aufeinander-

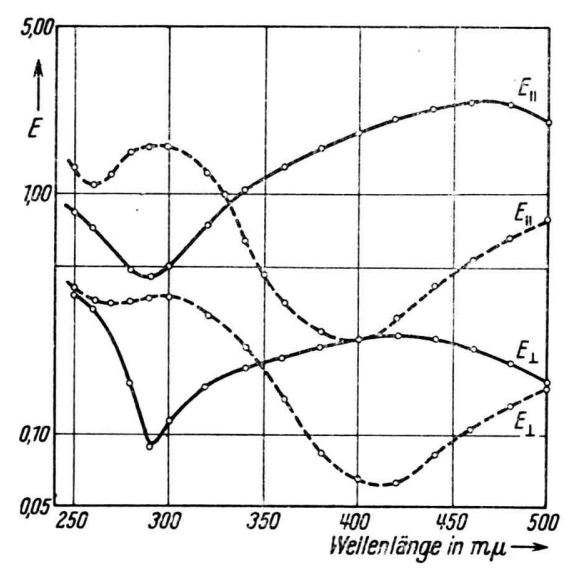

Abb. 4. Ausgezogene Kurve: Anfärbung mit Siriuslichtbraun G. Gestrichelte Kurve: Anfärbung mit Siriuslichtblau 3 RL. (Konz. der Farbbäder 0,5 g/100 cm ${ }^{3}$ ).

kitten und sie in dieser Form als Polarisator verwenden.

Günstiger ist jedoch, eine einzige Folie gleichzeitig mit einem Farbstoffgemisch anzufärben. Bringen wir z. B. die in den vorangegangenen Zeilen genannten Farbstoffe gemeinsam in Lösung, und zwar jede Komponente mit der gleichen Konzentration wie bei den Einzelanfärbungen, so resultieren Absorptionskurven, die sich aus den Absorptionswerten der Einzelanfärbungen berechnen lassen. Die $E_{\perp}$-Kurve dieser Anfärbung ist in Abb. 5 (Kurve 1) wiedergegeben. Kurve 2 ist aus $E_{\perp}$-Werten der Einzelfärbungen errechnet. Die Parallelverschiebung der Kurven kommt durch die verschiedene Dicke der Folien zustande. Bei gleichen Dicken würden die Kurven aufeinanderfallen.

Bei technischer Anwendung der Polarisationsfilter ist es oft ratsam, dieselben zwischen Schutzschichten zu kitten. Als solche können isotrope Kolloidfolien oder aber auch Glas- oder Quarzscheiben Verwendung finden. Darstellungen der Durchlässigkeitskurven sind denen der $E$-Kurven vorzuziehen. Abb. 6 gibt ein Beispiel für ein zwischen zwei Quarzplatten gekittetes Mischfarbenfilter. Es handelt sich um dasselbe Filter, dessen $E_{\text {, }}$-Kurve in Abb. 5 wiedergegeben war.

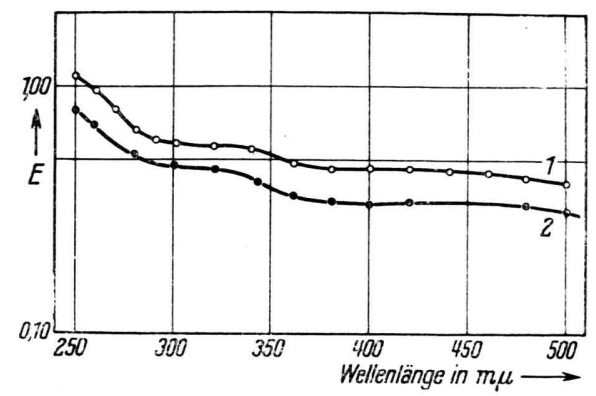

Abb. 5. $E_{\_}$-Kurve einer Anfärbung mit zwei Farbstoffen. (Siriuslichtbraun G und Siriuslichtblau 3 RL.) 1: gemessen; 2: errechnet aus den Werten der Abb. 4.

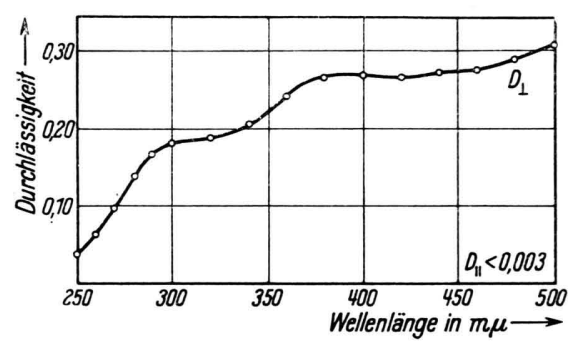

Abb. 6. Durchlässigkeit eines zwischen Quarzscheiben gekitteten Mischfarbenfilters.

\section{Dichroitische Metallfärbungen}

Wenn man lichtdurchlässige, linearorientierte hochpolymere Folien mit Metallsalzen anfärbt und später in der Folie die Metalle durch Reduktionsmittel zur Ausscheidung bringt, so ergeben sich häufig Produkte mit starkem Dichroismus. Abb. 7 zeigt die Absorptionskurven eines solchen dichroitischen Präparates, das durch Anfärbung von Silber an orientierten Filmen erhalten wurde.

Die bekannten Theorien ${ }^{1-3}$ sind nicht in der Lage, den Verlauf dieser Kurven näher zu erklären. Nach der B r a u n schen Gittertheorie müßte die den Gitterstäben parallel schwingende Komponente des Lichtes, also deren Lichtvektor $\mathfrak{E}$ parallel der Reckrichtung schwingt, maximale Absorption erleiden, während gerade an den Stellen höchster $E_{\|^{-}}$Werte die $E_{\perp}$-Werte nahezu verschwinden sollten. Das heißt, das zur Reckrichtung senkrecht schwingende Licht 
müßte im Spektralbereich der Absorptionsbande der $E_{\| \mid}$-Kurve gleichmäßig gut durchgelassen werden. Der Verlauf der $E$-Kurven bestätigt dies aber keineswegs, sondern spricht vielmehr für eine Verschiebung der Absorptionsbanden in den beiden ausgezeichneten Richtungen.

Auch die Ambronnsche Auffassung über die Ursache des Dichroismus versagt bei Anwendung auf diese Metallpolarisatoren vollkommen. Bekanntlich kristallisieren Metalle im regulären System, und diese liefern, in die Folie eingelagert, notwendig ein isotropes System.

Die $E$-Kurven der Abb. 7 werden dagegen verständlich, wenn wir die Adsorptionskräfte einer Betrachtung unterziehen. Dieselben gehen von den aktiven OH-Gruppen des Polyvinylalkohols oder des Cellulosehydrates aus. Durch die Reckung sind die kettenförmigen Moleküle orientiert, und diese Kräfte wirken senkrecht zur Reckrichtung. Bringt man nun Metallatome in die Folie,

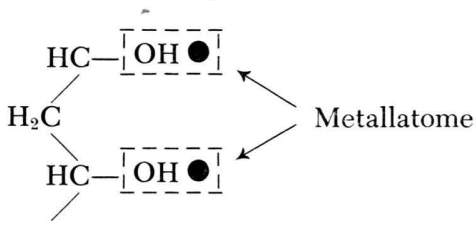

so unterliegen diese den von den $\mathrm{OH}-Z$ entren ausgehenden Kräften. Die Adsorption wird an den $\mathrm{OH}-G r u p p e n$ erfolgen und die Elektronenwolke der Metallatome eine Deformation erleiden, was sich optisch dann in einer anisotropen Lichtabsorption zeigt.

Dies kann mittels Potentialkurven erläutert werden. In Abb. 8 stellt die Kurve a-b-c die Änderung des Energieinhaltes des Systems (adsorbierende $\mathrm{OH}$ Gruppe plus adsorbiertes Metallatom) in Abhängigkeit vom Abstand dar, wenn das Atom sich im Grundzustand befindet. $A$ ist die Adsorptionsenergie. Die Kurve d-e-f ist die entsprechende Kurve für das angeregte Metallatom, unter der Annahme, daß $A^{\prime}$ kleiner ist als $A$ und außerdem der Abstand von der $\mathrm{OH}-\mathrm{Gruppe}$ im Gleichgewichtszustand für das angeregte Metallatom größer ist. Die Lichtabsorption durch das adsorbierte Atom entspricht dem Übergang von $b$ nach g. Das angeregte Atom nimmt dabei noch Schwingungsenergie auf (Schwingungen gegen die OH-Gruppe).

Im Falle der Abb. 8 erfordert die Lichtabsorption durch das Atom im adsorbierten Zustand mehr Energie als im freien:

$$
h v_{\text {ads. }}>\Delta E .
$$

Die Lichtabsorption erfolgt also bei kürzeren Wellen, d. h., wenn wir die Atome längs der Reckrichtung zunächst als frei annehmen, findet eine Verschiebung der Absorptionsbanden nach kürzeren Wellen für senkrecht zur Reckrichtung polarisiertes Licht statt. (Natürlich kann auch eine Verschiebung nach längeren Wellen erfolgen, das wäre der Fall, wenn $A^{\prime \prime}>A$.) Die Überlegungen bleiben auch noch stichhaltig, wenn

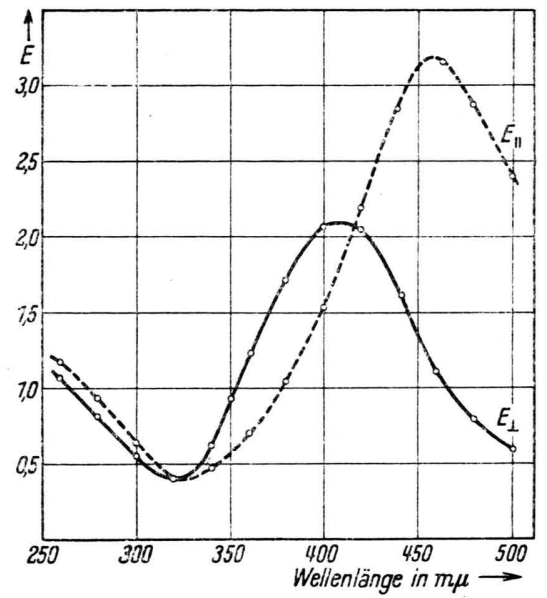

Abb. 7. Silbergefärbte Cellulosehydratfolie.

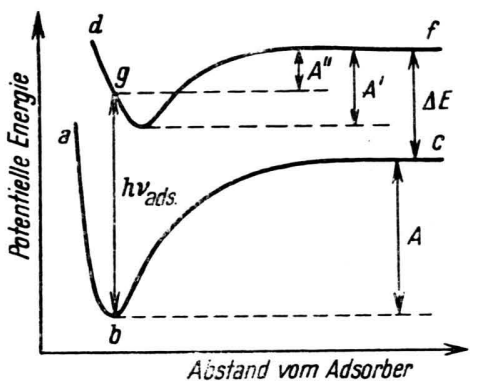

Abb. 8. Potentialverlauf bei der Adsorption.

die Metallatome in der Reckrichtung nicht als frei betrachtet werden dürfen. Für die Schwingung parallel zur Reckrichtung ergibt sich dann eine andere Potentialkurve als für die Schwingung senkrecht zur Reckrichtung und damit eine gegenseitige Verschiebung der Absorptionsbanden.

Die Abb. 7 zeigt aber nicht nur eine Verschiebung der Absorptionsbanden, sondern auch einen Unterschied in der Größe der Absorption. Dies läßt sich dadurch erklären, daß in der Adsorptionsrichtung Elektronenübergänge möglich werden oder an Übergangswahrscheinlichkeit gewinnen, die in der dazu senkrechten Richtung verboten sind bzw. eine ziem- 
lich kleine Übergangswahrscheinlichkeit besitzen und umgekehrt ${ }^{5,6}$.

Das Vorangehende läßt auch ohne weiteres den Dichroismus von mit Quecksilber angefärbten Folien verstehen, bei denen unter Normaltemperaturen bestimmt keine Kristalleinlagerung erfolgt.

\section{Jodpolaris atoren}

Anfärbungen von Jod an orientierten Polymerisaten von Vinylalkohol zeigen außerordentlich starken Dichroismus.

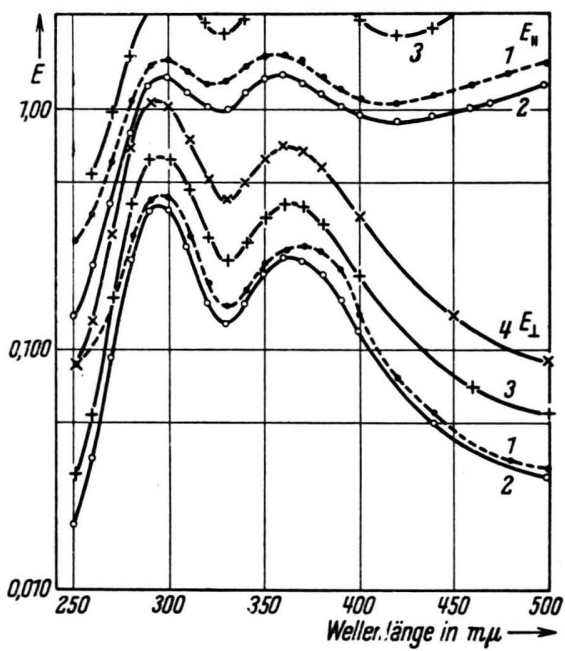

Abb. 9. Jodgefärbte Polyvinylalkoholfolien. Variiert wurde der KJ-Gehalt der Farbstofflösungen. 1. ohne KJ; 2. 0,05 $\mathrm{g} / 100 \mathrm{~cm}^{3} ; 3.0,12 \mathrm{~g} / 100 \mathrm{~cm}^{3} ; 4.0,25 \mathrm{~g} / 100 \mathrm{~cm}^{3}$.

In Abb. 9 sind die Absorptionskurven von jodgefärbten Polyvinylalkoholfolien wiedergegeben. Die jeweils mit gleichen Zahlen versehenen Kurven gehören zusammen.

Um diese Kurven deuten zu können, müssen wir auf Ergebnisse zurückgreifen, die de Boer und Custers ${ }^{7}$ bei der Untersuchung der Lichtabsorption von adsorbiertem Jod fanden. Sie ließen Jod an einer durch Sublimation im Hochvakuum entstandenen Calciumfluoridschicht adsorbieren. Dann werden bei sehr kleiner Oberflächenkonzentration nur die aktivsten Stellen der Oberfläche besetzt. Die Salzschicht hat in ihrer blättrigen Struktur zahlreiche sehr aktive Stellen.

Die elektrostatischen Felder dieser aktiven Steilen

5 H. Raus ch, v. Tra ube n berg, G. G e b a u e r u. G. L e w i n, Naturwiss. 18, 132 [1930].

${ }^{6} \mathrm{H} . \mathrm{K}$ u h n, Z. Physik 61, 805 [1930].

7 J. G. de B o e r u. J. F. H. Cus ters, Z. physik. Chem., Abt. B, 21, 208 [1933]. sind so stark, daß die elektrostatische Polarisation die Van der Waals'schen Adsorptionskräfte bei weitem überwiegt. Infolgedessen haben die an solchen Stellen adsorbierten Moleküle einen genau ausgerichteten induzierten Dipol.

Werden nur wenige Jodmoleküle adsorbiert, z. B. die Kurve $m / m_{0}=0,005$ in Abb. 10, dann kann die Bindung des Jods an die Oberfläche mit der des Jodmoleküls und Jodions im Trijodion verglichen werden $\left(J_{3}^{-}=J^{-} \cdot J_{2}\right)$. Bei größeren Werten ist die Bindung

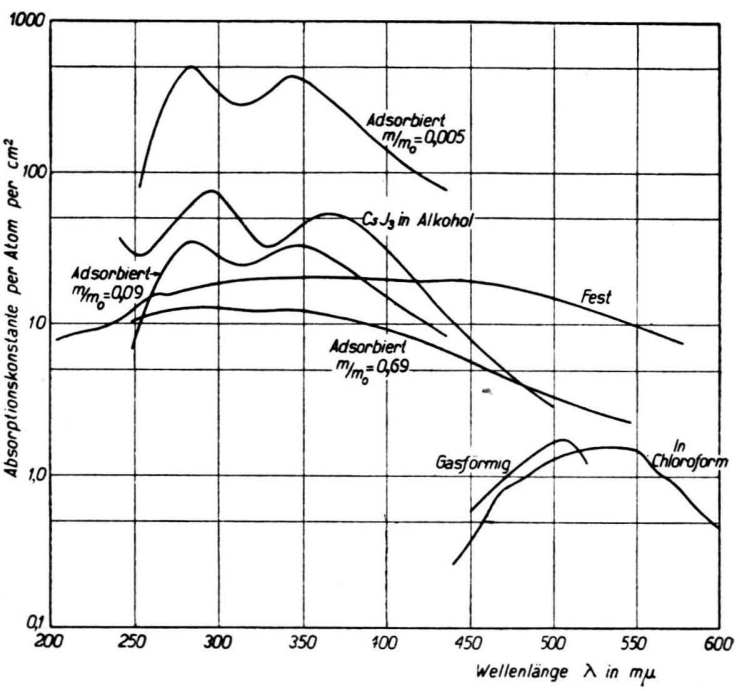

Abb. 10. Lichtabsorption von Jod bei verschiedener Bindung. $m / m_{0}=$ Bruchteil der Oberfläche, der von adsorbiertem Jod bedeckt ist. De Boer u. Custers ${ }^{7}$.

des Jodmoleküls, das jetzt nur mittels Van der Waals'scher Kräfte haftet, eher dem Zustand im festen Jod zu vergleichen, dessen Gitter ebenfalls nur mittels Van der Waals'scher Kräfte aufgebaut ist. Die Absorptionskurven der jodgefärbten Polyvinylalkoholfolien lassen sich dann folgendermaßen verstehen:

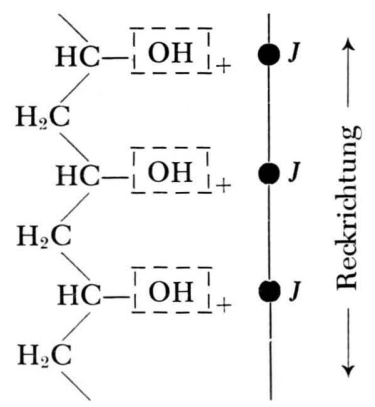

\footnotetext{
Schwingungsrichtung des $\leftleftarrows-$ Vektors $=\|$ Licht

$\longleftrightarrow$ Schwingungsrichtung des $\Subset$-Vektors $=\perp$ Licht
} 
Bei der Anfärbung setzen sich die Jodatome an die einzelnen aktiven OH-Gruppen. Hier werden durch die Adsorptionskräfte elektrostatischer Natur orientierte Dipole induziert, die senkrecht zur Reckrichtung der Folie liegen. Fällt jetzt Licht, dessen É-Vektor in dieser Richtung schwingt, auf das Filter, so wird die auf diese Weise gemessene Absorptionskurve $\left(E_{\perp}\right.$-Kurve) in ihrem Charakter den Kurven

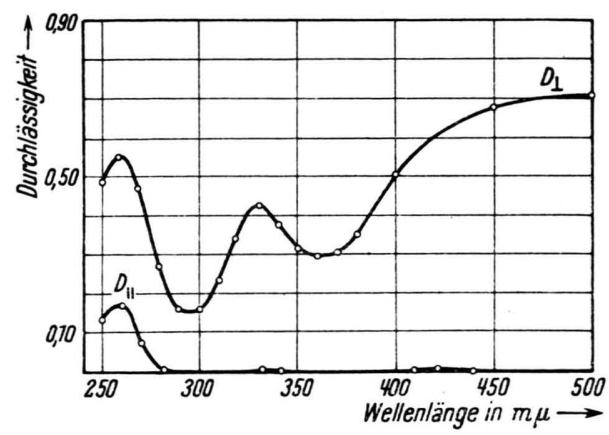

Abb. 11. Durchlässigkeit eines Jodfilters.

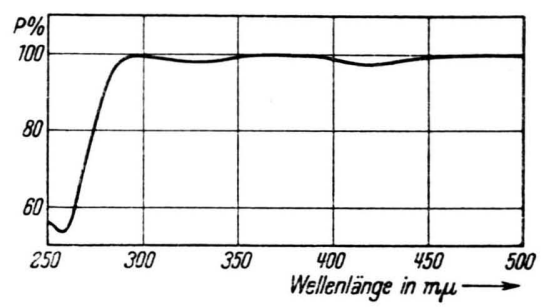

Abb. 12. Polarisationsgrad $P=\frac{D_{\perp}-D_{\| 1}}{D_{\perp}+D_{\|}}$eines Jodfilters.

gleichen, die sich bei Jod, adsorbiert an den aktiven Stellen des $\mathrm{CaF}_{2}$, ergaben (vgl. die $E_{\perp}$-Kurven der Abb. 9 mit der Kurve in Abb. 10 für $\left.m / m_{0}=0,005\right)$.

Für parallel der Reckrichtung polarisiertes Licht werden sich dagegen mehr die Van der Waals'schen Kräfte in der Absorption bemerkbar machen. Der Abstand der OH-Gruppen im Polyvinylalkoholmolekül beträgt nach Röntgenaufnahmen von $\mathrm{Halle}^{7} 2,57 \AA$. Adsorbierte Jodatome an diesen haben dann nahezu den gleichen Abstand wie im Jodmolekül (2,65 ̊̊). Die Absorption in dieser Richtung wird also mehr mole- kularer Natur sein, was sich durch den Anstieg der $E_{\| \mid}$-Werte nach längeren Wellen zeigt. Dieses auftretende Maximum findet sich bei allen Absorptionskurven, bei denen Jod in molekularer Form als $\mathrm{J}_{2}$ vorhanden war. (Siehe z. B. die Kurven "gasförmig" und "Chloroform gelöst" in Abb. 10.)

Zum Schluß seien die Durchlässigkeit (Abb. 11) und der Polarisationsgrad (Abb. 12) eines jodgefärbten Polarisationsfilters wiedergegeben. Die Absorption des ungekitteten Filters zeigt Abb. 9 (Kurve 3).

\section{Zusammenfassendes Ergebnis}

1. Alle dichroitischen Erscheinungen an gefärbten orientierten Folien lassen sich erklären, wenn man die Adsorption der Farbstoffe berücksichtigt.

2. Bei Adsorption eines Atoms oder Moleküls an aktiven Stellen (z. B. den OH-Gruppen) zeigt sich, daß das System anisotrop wird. Die Einwirkung auf das Atom oder Molekül ist unsymmetrisch. Es ist deshalb wahrscheinlich, daß seine Eigenschaften in der Richtung, in der die Adsorptionskräfte wirken, anders sind als senkrecht dazu. (Beispiele sind die Metall- und Jodfärbungen.)

3. Haben die adsorbierten Moleküle oder Molekülaggregate von Natur aus eine optische Anisotropie, so bewirken die Adsorptionskräfte eine gemeinsame Orientierung dieser Moleküle. Das Ergebnis sind $E_{\|^{-}}$und $E_{\perp}$-Kurven, die durch den Charakter des Molekülbaues der Farbstoffe bestimmt werden. (Dies Verhalten zeigen oft die organischen Farbstoffe.)

4. Wie die Abb. 6, 11 und $13^{*}$ zeigen, ist es ohne weiteres möglich, Farbstoffpolarisatoren auch für ultraviolettes Licht zu bauen.

Herrn Prof. Dr. W. H a n l e, in dessen Instiiıt die vorliegenden Untersuchungen ausgeführt wurden, bin ich für die Anregung zu dieser Arbeit, für sehr wertvolle Hinweise bei ihrer Ausführung sowie für sein großes Entgegenkommen zu Dank verpflichtet. Ferner habe ich Herrn Prof. Dr. P. Ce rmak sowie Herrn Prof. Dr. W. S chmidt für anregende Diskussionen und Hilfe und der Notgemeinschaft der Deutschen Wissen$\mathrm{schaft}$ für Überlassung von Apparaten bestens zu danken.

* Abb. 13, s. Tafel S. 440 b. 